\title{
The association between HERG gene expression and cardiac arrhythmia disease in children
}

\author{
MINGYU FU, CHUNLI LI, QINGWEN WANG, YAN WANG and XINJIANG AN \\ Department of Cardiology, Xuzhou Children's Hospital, Xuzhou, Jiangsu 221002, P.R. China
}

Received April 15,2016; Accepted September 28, 2016

DOI: $10.3892 /$ etm.2016.3802

\begin{abstract}
We explored the possible link between the expression of HERG gene and cardiomyopathy in children. From April 2013 to April 2015, 73 children with cardiac arrhythmia who were treated were enrolled in the present study to serve as the observation group. At the same time, 76 normal individuals were also enrolled as the control group. HERG expression level in the observation group was compared with the control group. To determine the level of HERG gene expression we used fluorescent directional PCR, enzyme immunoassay and western blot analysis. The results showed that HERG mRNA level in the observation group was significantly higher than that of the control group. The level of HERG protein in the observation group was significantly higher as well. In the observation group, HERG expression gradually increased with time during the course of the disease. This result suggested that $H E R G$ gene expression was associated with the severity of cardiac arrhythmia in children. HERG expression may be the cause of deterioration in cardiomyopathy. The results have provided a theoretical and practical basis for the diagnosis and treatment of children cardiomyopathy. Thus, we established a correlation between HERG expression and cardiac arrhythmia in children.
\end{abstract}

\section{Introduction}

Any changes in physiological and biochemical status during childhood can lead to cardiac arrhythmia in children. These include heart-related myocardial cell excitability and automaticity and conductivity (1). Possible causes of cardiac arrhythmia in children are complex (2). This disease may be caused by congenital factors or be induced by certain acquired diseases, including myocarditis, rheumatic fever, toxins, drug side effects and cardiac surgery sequelae (3). Arrhythmia

Correspondence to: Dr Xinjiang An, Department of Cardiology, Xuzhou Children's Hospital, 18 Sudibei Road, Xuzhou, Jiangsu 221002, P.R. China

E-mail: anxinjian001@163.com

Key words: HERG gene, cardiac arrhythmia of children, RT-PCR, enzyme-linked immunosorbent assay, correlation fatigue, nervousness and imperfect autonomic nervous function of children can induce myocarditis and congenital heart disease (4).

Poisoning caused by sympathomimetic amines and quinidine, acid-base balance disorders and electrolyte imbalance may lead to heart rate imbalances in children and may even deteriorate their condition (5). Due to the fact that heart rate imbalance in children is caused by complex factors, there is no specific therapeutic drug available (6). Results from a prior study revealed that the current treatment of heart rate imbalance is based on distinguishing heart rate imbalance symptoms using the frequency of premature beats and if the frequency of premature beats were reduced or disappeared after less activity, drug treatment would not be needed (7). Patients with long-standing symptoms of premature beat and diversity on electrocardiogram (ECG) can be treated by taking propafenone or propranolol $\beta$-blockers. However, there are studies showing that these drugs have no significant effects on heart rate imbalance symptoms of children (8).

The HERG gene product is known to be involved in cellular and molecular mechanisms controlling the metastasis and regulating the excitation of nerve and muscle cells (9). However, to the best of our knowledge, no study on the possible role of HERG in children's heart rate imbalance has been previously conducted. We explored the possible association between HERG expression and the heart rate imbalance of children and provide certain theoretical and experimental bases for rapid diagnosis and treatment of heart rate imbalances in children.

\section{Materials and methods}

General data. From April 2013 to April 2015, 73 cases of children with cardiac arrhythmia treated at the Xuzhou Children's Hospital (Jiangsu, China) were enrolled in this study to serve as the observation group. At the same time, 76 normal individuals were enrolled as the control group. The observation group comprised 38 males and 35 females with an average age of $6.2 \pm 3.8$ years. The control group had 36 males and 40 females with an average age of $5.8 \pm 3.2$ years. All participants were evaluated in accordance with the relevant standards and tests from ECG of children with cardiomyopathy and showed no signs of any related diseases. The study was approved by the Ethics Committe of Xuzhou Children's Hospital. Written informed consent was obtained from the parents of all the participants prior to the start of the study. 
Samples. We collected $5 \mathrm{ml}$ venous blood from all the cases and centrifuged samples at $2,500 \mathrm{x}$ g for $10 \mathrm{~min}$ at $4^{\circ} \mathrm{C}$ to separate the serum, and the samples were stored at $-80^{\circ} \mathrm{C}$. Freeze-stored solution was added to the remaining cells, and the cells were cryopreserved at $-80^{\circ} \mathrm{C}$ for follow-up experiments. HERG antibody used in the present study was purchased from Roche Diagnostics (Basel, Switzerland), RNA extraction kits were purchased from Axygen Biotechnology Co., Ltd. (Silicon Valley, CA, USA), the associated molecular reagents were purchased from Takara Biotechnology, Co., Ltd. (Dalian, China) and the fluorescence quantitative polymerase chain reaction (PCR) primers were designed by Primer 5 software and synthesized by Shanghai Sangon Biological Engineering Co. Ltd. (Shanghai, China).

\section{$R T-P C R$}

RNA extraction. For RNA extraction, we followed the standard protocol of Axygen kit (10), and the specific schemes were as follows: i) $0.6 \mathrm{ml}$ of RNA Plus was added to $0.2 \mathrm{~g}$ of frozen cell sample $\left(-80^{\circ} \mathrm{C}\right)$ and the mix was transferred to a precooled mortar and pestle. The sample was then moved into a $1.5 \mathrm{ml}$ sterilized EP tube. The mortar was washed with $0.3 \mathrm{ml}$ of RNA Plus and the liquid was added to the EP tube; ii) chloroform $(200 \mu \mathrm{l})$ was added and the tube was agitated for $15 \mathrm{sec}$ and left at room temperature for $15 \mathrm{~min}$; iii) the sample was centrifuged at $10,000 \mathrm{x}$ g for $15 \mathrm{~min}$ at $4^{\circ} \mathrm{C}$; iv) supernatant was transferred into EP tube with RNase and then isovolumetric isopropanol was added. After mixing, tube was incubated at room temperature for $10 \mathrm{~min}$; v) samples were centrifuged again $\left(10,000 \mathrm{x}\right.$ g at $4^{\circ} \mathrm{C}$ for $\left.10 \mathrm{~min}\right)$; vi) the supernatant was discarded and $750 \mu \mathrm{l}$ of $75 \%$ ethanol was added to the pellet and mixed gently. It was then centrifuged at $10,000 \mathrm{x} \mathrm{g}$ for $10 \mathrm{~min}$ at $4^{\circ} \mathrm{C}$; vii) supernatant was discarded and the residual ethanol was removed; and viii) appropriate amount of water was added to remove the RNase. The quality of the extracted RNA was evaluated according to a previous report (11).

Fluorescence quota PCR. Fluorescence quota PCR kit was purchased from Takara Biotechnology, Co., Ltd. Three-step method was used according to the instructions provided by the manufacturer's manual. Primer sequences are shown in Table I.

Enzyme-linked immunosorbent assay (ELISA). ELISA was used to verify the level of HERG protein in the serum. We followed a standard ELISA protocol (12):

i) Coating: $100 \mu \mathrm{l}$ of diluted HERG primary antibody $(5-10 \mu \mathrm{g} / \mathrm{ml})$ was added into a 96-well plate and incubated at $4^{\circ} \mathrm{C}$ overnight and solution was discarded the following morning. Wells were then washed with washing buffer solution provided in the kit (5 washes and $1 \mathrm{~min} / \mathrm{wash}$ ).

ii) Samples: $100 \mu \mathrm{l}$ of sample was added to each well and plate was incubated at $37^{\circ} \mathrm{C}$ for $1 \mathrm{~h}$. The plate was then washed (5 times, $1 \mathrm{~min} /$ wash).

iii) Secondary antibody: $100 \mu \mathrm{l}$ of second antibody was added and incubated at $37^{\circ} \mathrm{C}$ for $30-80 \mathrm{~min}$. The plate was then washed with washing buffer solution (5 times, $2 \mathrm{~min} /$ wash).

iv) Chromogenic substrate: $100 \mu \mathrm{l}$ of the freshly-prepared chromogenic substrate solution was added followed by incubation at $37^{\circ} \mathrm{C}$ for $30 \mathrm{~min}$.
Table I. PCR primer sequences.

\begin{tabular}{lcc}
\hline Gene & Primer sequence & $\begin{array}{c}\text { Fragment } \\
\text { length (bp) }\end{array}$ \\
\hline HERG & F: 5'-TGACGTAGTCAAGTACGGTAG-3' & 128 \\
& R: 5'-TGCGTATTGCCGTAGCTGCGGC-3' & \\
GAPDH F: 5'-GAAGGTGAAGGTCGGAGTC-3' & 226 \\
& R: 5'-GAAGATGGTGATGGGATTTC-3' & \\
&
\end{tabular}

PCR, polymerase chain reaction; F, forward; $\mathrm{R}$, reverse.

v) Stop buffer: $20 \mu 1$ of $0.2 \mathrm{M}$ sulfuric acid was added to stop the reaction.

vi) Qualitative and quantitative tests: For qualitative observation, a plate was placed on a blank paper and color depth was compared. For the quantitative test, we used a microplate reader (Bio-Rad, Hercules, CA, USA) which was set at $450 \mathrm{~nm}$.

Western blot analysis. We used the standard protocol for western blot analysis (all the antibodies were purchased from Roche Diagnostics).

Immunohistochemical detection of AVRI in myocardial tissue. In the present study, we conducted the conventional antibody incubation and staining in cardiomyopathy tissue samples by streptomycin and enzymes (S-P). The immunohistochemical standard protocol was as follows (9): Membrane staining $<10 \%$ or cardiomyocytes after staining presenting negative was considered as negative. Membrane staining or the membrane staining of $>10 \%$ of myocardial cells was considered positive (+). When $>10 \%$ of myocardial cells showed weak or moderate staining we considered it as (++). When $>10 \%$ of myocardial cells were showing strong complete membrane staining, we considered it as (+++).

Data processing. SPSS 20.2 statistical software (Chicago, IL, USA) was used for the statistical analysis. Measureable data were presented as mean \pm SD and categorical data were analyzed using the Chi-square test. $\mathrm{P}<0.05$ was considered to indicate a statistically significant difference.

\section{Results}

HERG transcription level. Results obtained from the fluorescence quantitative PCR showed that the level of HERG mRNA was considerably higher in the observation group compared to the control group (Fig. 1). The difference was statistically significant $(\mathrm{P}<0.05)$. These results showed that $H E R G$ gene was actively transcribed in children with cardiomyopathy.

HERG protein expression measured by ELISA. Results obtained from ELISA showed that the level of HERG protein expression was markedly stronger in the observation group compared to the control group (Fig. 2). The difference was statistically significant $(\mathrm{P}<0.05)$.

These results were in accordance with fluorescence quantitative PCR results showing higher levels of HERG expression in children suffering from cardiomyopathy. 


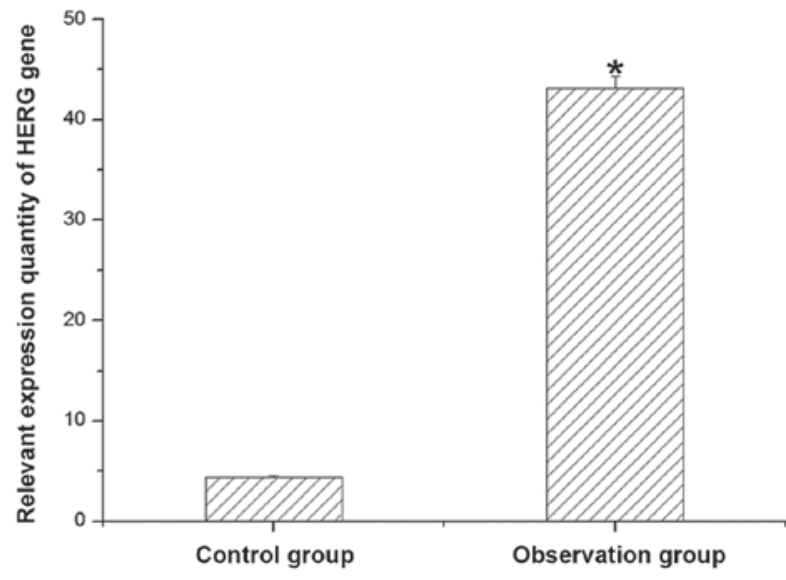

Figure 1. HERG mRNA level. ${ }^{*} \mathrm{P}<0.05$.

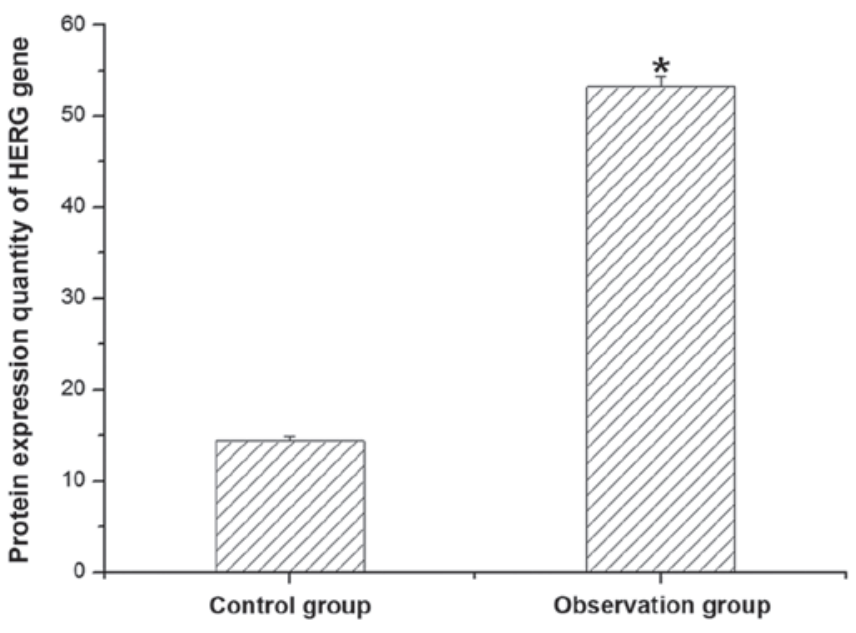

Figure 2. HERG protein expression measured by enzyme-linked immunosorbent assay (ELISA). ${ }^{*} \mathrm{P}<0.05$.

HERG protein expression measured by western blot analysis. To verify and confirm the results obtained from ELISA, a western blot analysis was conducted on samples. The results obtained from the western blot analysis showed that the level of HERG protein expression was markedly stronger in the observation group compared to the control group (Fig. 3). The difference was statistically significant $(\mathrm{P}<0.05)$. This was in accordance with the ELISA results and confirmed that

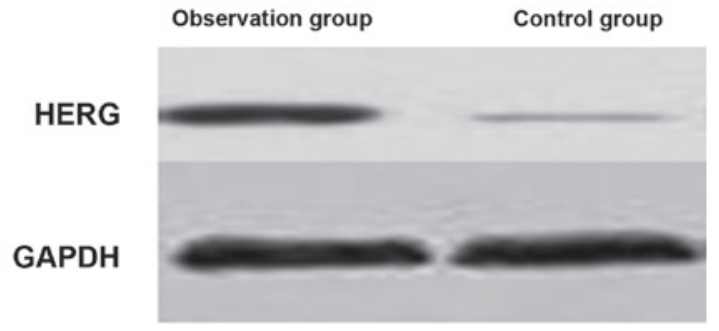

Figure 3. HERG protein expression levels measured by western blot analysis.

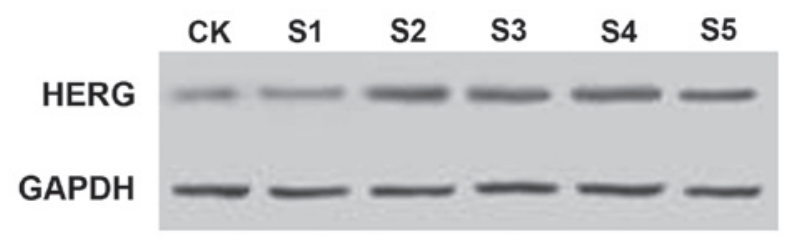

Figure 4. HERG protein level in the serum at different times in the course of the disease in the observation group. CK, the control group; S1-S5, the observation group in $3,16,28,36,45$ and 52 months.

relatively higher amounts of HERG protein were present in in children suffering from cardiomyopathy.

To examine the association between the expression level of HERG and the condition of children with cardiomyopathy, we measured HERG expression in the serum of the ill children in the observation group at different times of the disease (Fig. 4). The results showed that in the observation group, HERG expression gradually increased during the course of the disease. After a certain time in the course of the disease the HERG expression decreased indicating that HERG can suppress the deterioration of children's codition to some extent.

HERG in myocardial tissue tested by immunohistochemistry. The immunohistochemistry results showed that the positive staining was mainly concentrated in myocardial cell membrane in sick children from the observation group.

Cardiac arrhythmia of children (Fig. 5). This suggested that the HERG level in children with cardiomyopathy was higher than the normal control group.

\section{Discussion}

As a common pediatric clinical disease with very complex causes, cardiac arrhythmia of children can be caused by
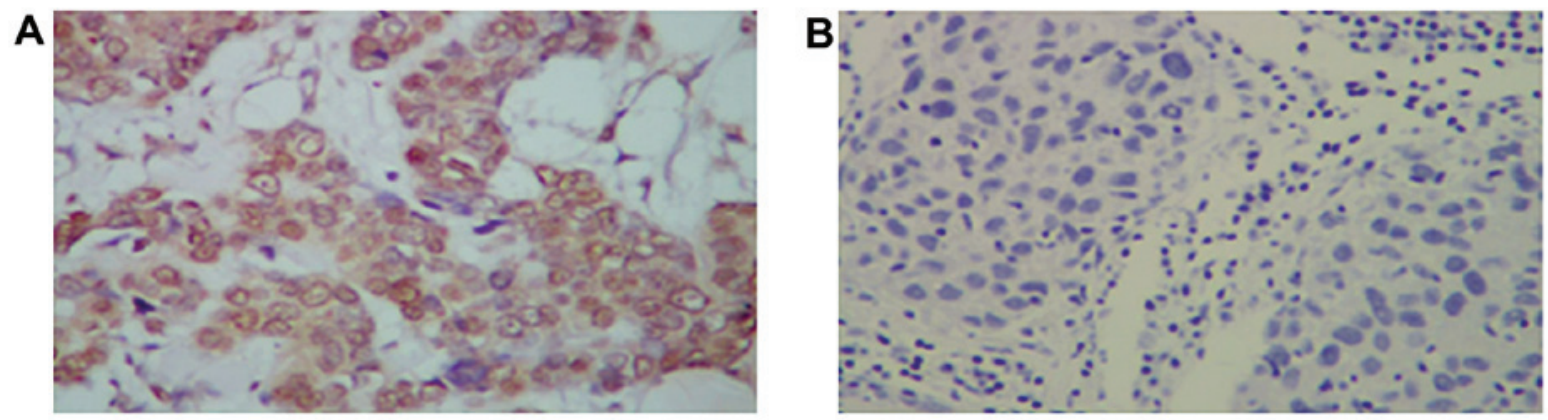

Figure 5. HERG protein in myocardial tissue tested by immunohistochemistry. (A) The observation group; (B) the control group. 
pediatric heart disease and non-cardiac diseases, such as hypoxia, and electrolyte imbalance to some extent. Therefore, the proper diagnosis and treatment for arrhythmia of children have become an important issue. Brembilla-Perrot et al (13) showed that the current diagnosis for arrhythmia in children is mainly achieved through ECG. There are some complications associated with ECG diagnostics, therefore the diagnosis of cardiac arrhythmias in children of different regions in China is not easy (14). There is still no specific medication for treating arrhythmia disease of children. According to Bian et al (15), HERG protein expression is linked to regulation of neural cells in human body (16) as well as the expression of potassium channel membrane proteins.

Results from a study by Phartiyal et al (17) showed higher levels of HERG in human gastric cancer tissues. Significantly lower levels of HERG have been reported in necrotic muscle cells $(18,19)$. This result indicates that HERG may be involved in the improvement of muscle cell excitability (20).

In the present study, from the measured HERG expression it was demonstrated that HERG expression in children suffering from cardiac arrhythmia increased significantly.

In the course of the disease, HERG expression increased gradually, suggesting that there was a correlation between HERG expression and arrhythmia disease in children. It also suggested that the expression of HERG may cause the arrhythmia or help its deterioration. Our results provided a theoretical and experimental basis for cardiac arrhythmia disease research in children.

\section{References}

1. Murayama H, Maeda M, Sakurai H, Usui A and Ueda Y: Predictors affecting durability of epicardial pacemaker leads in pediatric patients. J Thorac Cardiovasc Surg 135: 361-366, 2008.

2. Li L and Yue Y: Evidence-based medicine evaluation on pediatric antiarrhythmic drugs. J Applied Clin Pediatrics 27: 263-367, 2012 (In Chinese).

3. Xiaodong Y: Clinical diagnosis and therapy on cardiac arrhythmia of children. Chin J Clin Physicians 2012: 7961-7964, 2012 (In Chinese).

4. Schwartz PJ: Cascades or waterfalls, the cataracts of genetic screening are being opened on clinical cardiology. J Am Coll Cardiol 55: 2577-2579, 2010.

5. Juheng Y, Guohong Z and Ying X: Guidance from CARTO on the radiofrequency ablation treatment of cardiac arrhythmia of children. J Clin Pediatrics 1: 389-392, 2011.
6. Fen L: Pharmacotherapy progresses on cardiac arrhythmia of children. J Pediatric Pharmacy 5: 389-392, 2012.

7. Kriebel T, Saul JP, Schneider H, Sigler M and Paul T: Noncontact mapping and radiofrequency catheter ablation of fast and hemodynamically unstable ventricular tachycardia after surgical repair of tetralogy of Fallot. J Am Coll Cardiol 50: 2162-2168, 2007.

8. Lee PC, Hwang B, Chen SA, Tai CG, Chen YJ, Chiang CE and Meng CC: The results of radiofrequency catheter ablation of supraventricular tachycardia in children. Pacing Clin Electrophysiol 30: 655-661, 2007.

9. Chencheng D, Baojing G and Ling H: 164 cases of clinical analysis and ablation effect of children's right side. J Clin Electrocardiol 2014: 43-46, 2014.

10. Li BX, Yang BF, Zhou J, Xu CQ and Li YR: Inhibitory effects of berberine on IK1, IK, and HERG channels of cardiac myocytes. Acta Pharmacol Sin 22: 125-131, 2001.

11. Liu J, Zhang M, Jiang M and Tseng GN: Structural and functional role of the extracellular s5-p linker in the HERG potassium channel. J Gen Physiol 120: 723-737, 2002.

12. Dai C, Wei S, Guo B and Han L: One case on the remission treatment for of persistent and complete LBBB after VSDO. Chinese J Interventional Cardiol 6: 358-359, 2010 (In Chinese).

13. Brembilla-Perrot B, Cloez JL, Marchal C, Chometon F, Huttin O, Tatar C, Lethor JP, Tisserand A, Admant P, Belhadj K, et al: Transesophageal electrophysiological study in non sedated children younger than 11 years with a Wolff-Parkinson-White syndrome. Ann Cardiol Angeiol (Paris) 58: 1-6, 2009 (In French).

14. Kang J, Wang L, Chen XL, Triggle DJ and Rampe D: Interactions of a series of fluoroquinolone antibacterial drugs with the human cardiac $\mathrm{K}^{+}$channel HERG. Mol Pharmacol 59: 122-126, 2001

15. Bian JS, Kagan A and McDonald TV: Molecular analysis of PIP2 regulation of HERG and IKr. Am J Physiol Heart Circ Physiol 287: H2154-H2163, 2004.

16. Sujuan K, Jijin L and Min Y: Gene transfection of HERG on HEK293 cells and record of its coding passcurrent. Chin J Card Pacemak ECG 2008: 255-258, 2008.

17. Phartiyal P, Jones EM and Robertson GA: Heteromeric assembly of human ether-à-go-go-related gene (hERG) 1a/1b channels occurs cotranslationally via $\mathrm{N}$-terminal interactions. J Biol Chem 282: 9874-9882, 2007.

18. Thomas D, Kiehn J, Katus HA and Karle CA: Adrenergic regulation of the rapid component of the cardiac delayed rectifier potassium current, $\mathrm{I}(\mathrm{Kr})$, and the underlying $\mathrm{hERG}$ ion channel. Basic Res Cardiol 99: 279-287, 2004.

19. Zhang H, Zou B, Yu H, Moretti A, Wang X, Yan W, Babcock JJ, Bellin M, McManus OB, Tomaselli G, et al: Modulation of hERG potassium channel gating normalizes action potential duration prolonged by dysfunctional KCNQ1 potassium channel. Proc Natl Acad Sci USA 109: 11866-11871, 2012.

20. Gong Q, Jones MA and Zhou Z: Mechanisms of pharmacological rescue of trafficking-defective hERG mutant channels in human long QT syndrome. J Biol Chem 281: 4069-4074, 2006. 